\title{
Colchicine-an old dog with new tricks
}

\author{
Colchicine is an anti-inflammatory agent that suppresses myeloid cell activation and is commonly used to treat \\ gout. In this issue of Nature Metabolism, Weng et al. demonstrate that colchicine exerts its anti-inflammatory \\ effects in mice via the induction of the hormone GDF-15 in the liver.
}

\section{Samuel M. Lockhart and Stephen O'Rahilly}

olchicine is an inexpensive, widely available drug with well-known anti-inflammatory activity. It is commonly used for the treatment of acute gout, a crystal arthritis associated with severe pain and a systemic inflammatory response. In recent years, colchicine has enjoyed somewhat of a renaissance, after high-quality randomized controlled trials demonstrated its effectiveness in the treatment of pericarditis ${ }^{1}$ and in the secondary prevention of atherosclerotic cardiovascular disease ${ }^{2}$. The therapeutic utility of colchicine in treating COVID19 is being tested in ongoing randomized controlled clinical trials, and a recent preprint of a randomized controlled trial of colchicine in outpatients with COVID-19 has reported a decrease in hospitalization in those with polymerase chain reactionconfirmed COVID-19 (ref. ${ }^{3}$ ). The anti-inflammatory action of colchicine is commonly accepted to depend on a direct effect of colchicine in myeloid cells. However, in this issue of Nature Metabolism, Weng et al. ${ }^{4}$ uncover a novel mechanism of action of colchicine that depends on the secretion of the hormone GDF-15 from the liver.

Weng et al. astutely observed that the doses of colchicine required to inhibit myeloid cell chemotaxis and inflammasome activation in previous studies are much lower than those achieved with therapeutic doses in humans, and therefore hypothesized that colchicine's action on myeloid cells might be indirect. To test this hypothesis, they treated primary mouse neutrophils ex vivo with plasma from colchicine-treated mice or vehicle-treated mice. In support of their hypothesis, plasma from colchicine-treated mice, but not vehicle-treated mice or vehicle-treated mice with ex vivo addition of colchicine, impaired neutrophil activation. To identify the site of action of colchicine and the source of the secreted factor, the authors used activation of Jun kinase as a bioassay to detect colchicine activity and demonstrated that colchicine was selectively

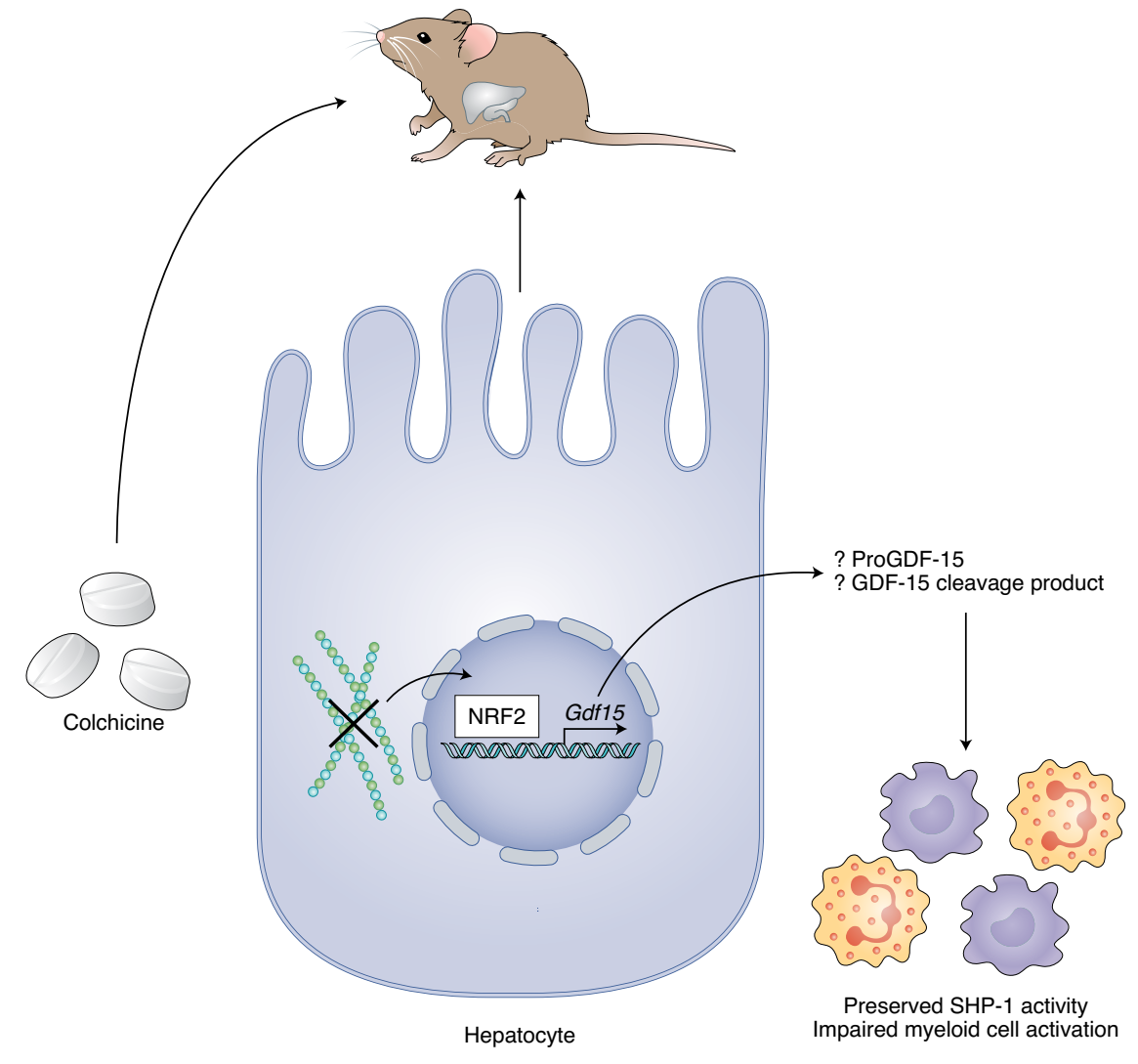

Fig. 1 | Colchicine induces hepatic GDF-15, which in turn suppresses inflammation by inhibiting myeloid cell activation. Colchicine selectively depolymerizes tubulin in hepatocytes, thus inducing Gdf15 gene expression via an NRF2-dependent mechanism. An unknown form of GDF-15 that differs from the mature peptide is secreted and acts on myeloid cells through an unknown receptor, thereby preventing inhibitory SHP-1 phosphorylation, impairing macrophage and neutrophil activation, and suppressing inflammation.

active in hepatocytes at therapeutic doses in vivo. This finding, as the authors note, is consistent with the known role of hepatocytes in xenobiotic metabolism.

GDF-15 is released by cells under a variety of stressful conditions. It signals via a brainstem-restricted receptor, a GFRAL-Ret heterodimer, thus resulting in a variety of responses that help protect the organism in the short term and promote future avoidance of the stress-initiating agent ${ }^{5}$. Using existing gene expression datasets, the authors identified GDF-15 as a potential candidate mediator of the effects of colchicine. In agreement with a role of GDF-15, colchicine treatment in mice induced GDF-15 in the liver via a mechanism dependent on the transcription factor Nrf2 and increased circulating GDF-15 with kinetics consistent with the anti-inflammatory action of colchicine. Moreover, liver-specific short interfering 
RNA-mediated knockdown of GDF-15, GDF-15 neutralization with an antibody to GDF-15 and germline deletion of GDF-15 all ablated the anti-inflammatory action of colchicine in two separate mouse models of acute inflammation. Ex vivo, the inhibitory effects of plasma from colchicine-treated mice on myeloid activation were not observed with plasma from $G d f 15^{-/-}$mice or mice treated with hepatocyte-specific Gdf15 short interfering RNA. To demonstrate a direct anti-inflammatory effect of GDF-15, the authors blocked GDF-15 in the plasma by using three different antibodies to GDF-15. Treatment with each antibody normalized myeloid cell activation, thus rigorously demonstrating that an immunoreactive GDF-15 moiety must be active in plasma to mediate the anti-inflammatory effects of colchicine. The authors also demonstrate that GDF15-containing plasma blocks the inhibitory phosphorylation of the phosphatase SHP-1 in myeloid cells in a GDF-15-dependent manner and that SHP-1 inhibition in vivo ablates the anti-inflammatory actions of colchicine in mice.

Thus, colchicine impairs myeloid cell activation in mice by inducing hepatic GDF15 secretion, but how GDF-15 mediates this anti-inflammatory activity remains unclear. The anti-inflammatory effects of GDF-15 were observed in mice lacking the canonical GDF-15 receptor, GFRAL. Moreover, mature recombinant GDF-15 did not exhibit anti-inflammatory action in mice in vivo or in myeloid cells treated ex vivo. Thus, a product of the Gdf15 gene, which has epitopes recognized by existing antibodies to GDF-15 but is not the mature GDF15 disulfide-linked dimer itself, appears to exert anti-inflammatory action on myeloid cells via a receptor that has yet to be identified.

Therefore, although an intriguing observation has been made, gaining comprehensive understanding will be challenging and will actually require two big leaps. First, what is the nature of the GDF15-related moiety that causes this effect on neutrophils? Second, what receptor is it acting through?

Gdf15 mRNA is translated to a $308-$ amino acid pre-proGDF-15 with signal and propeptide domains. Monomeric proGDF-15 forms a disulfide bond with a second proGDF-15 monomer in the endoplasmic reticulum. Dimeric proGDF-15 is then cleaved by endogenous proteases and forms mature, dimeric GDF-15, which is understood to be the major circulating form. Whether proGDF-15 circulates is unclear, but it can be secreted from transformed cell lines ${ }^{5}$. Could proGDF-15 be the mysterious anti-inflammatory GDF-15 moiety described here? Interestingly, proGDF-15, but not mature GDF-15, secreted from prostate cancer cell lines can bind the extracellular matrix, thus suggesting that the proprotein form has specific binding ability distinct from that of mature GDF15 (ref. ${ }^{6}$ ). It is intriguing to speculate that extracellular-matrix-bound GDF-15 might represent a pool of anti-inflammatory peptide to be released by tissue destruction. In addition, MMP14 has been reported to cleave mature GDF-15, producing a 6-kilodalton carboxy-terminal fragment, although the biological importance of either cleavage product is unclear ${ }^{7}$. Another formal possibility is that post-translational modification of GDF15 may enable it to exert its effects on neutrophils, thus potentially explaining why recombinant GDF15 is inactive in that regard.

How might a molecule containing the core GDF-15 exhibit anti-inflammatory action on myeloid cells? Importantly, the selectivity of mature GDF-15 for GFRAL has been rigorously demonstrated. Two groups have independently screened more than 4,000 membrane-bound proteins for GDF-15 binding, and a third group has screened all single-pass transmembrane receptors ${ }^{5}$. All three groups found extremely high specificity for the interaction of GDF15 with GFRAL. Robust and replicated evidence for GFRAL expression anywhere other than the brainstem is difficult to identify. In the case of the colchicine work, however, we must look beyond GFRAL, because the anti-inflammatory effects persisted in its absence. To explain these findings, we must assume the existence of a myeloid-expressed receptor distinct from GFRAL. Until the GDF15-related molecule is identified, definitively identifying the receptor mediating its action will be challenging. That said, high-throughput screens in myeloid cells by using plasma from colchicine-treated animals as the stimulus and SHP-1 phosphorylation as the readout might enable the identification of a candidate receptor. This finding could then be interrogated with a broad range of synthetic or purified forms of proGDF15 or its processed forms.

The findings of Weng et al. (Fig. 1) raise broader issues regarding GDF15's biology. The hindbrain-restricted nature of the GFRAL receptor has called into question reports of a multitude of peripheral actions attributed to GDF-15 before the discovery of GFRAL. Some of these actions may be explained by contamination of recombinant GDF-15 with TGF $\beta$ (ref. ${ }^{8}$ ), but several laboratories have described peripheral actions of GDF-15 through genetic manipulation of the hormone, which remain unexplained. For example, $G d f 15^{-/-}$mice exhibit increased myocardial inflammation in experimental models of myocardial infarction ${ }^{9}$ and, in agreement with an inhibitory effect of GDF-15 on myeloid cell recruitment, $\mathrm{Gdf} 15^{-/-}$mice exhibit enhanced neutrophil recruitment in experimental models of sepsis ${ }^{10}$. Although these effects of GDF15 could be mediated indirectly via autonomic or neuroendocrine outputs of the brain, initiated by GFRAL-Ret signalling in the medulla, the findings of Weng et al., which demonstrate the existence of a peripherally expressed receptor transducing an anti-inflammatory signal for a Gdf15-encoded molecule that is not mature GDF15, provide an alternative explanation for these puzzling findings. It is a truism that interesting research raises more questions than it answers. In this case, this statement is genuinely true.

\section{Samuel M. Lockhart (D)1 and Stephen O'Rahilly (1D)}

${ }^{1}$ MRC Metabolic Diseases Unit, Wellcome Trust-Medical Research Council Institute of Metabolic Science, University of Cambridge, Cambridge, UK. ${ }^{2} M R C$ Metabolic Diseases Unit, Wellcome Trust-Medical Research Council Institute of Metabolic Science, University of Cambridge,

Cambridge, UK.

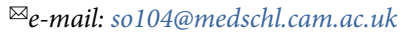

Published online: 12 April 2021 https://doi.org/10.1038/s42255-021-00360-4

References

1. Imazio, M. et al. N. Engl. J. Med. 369, 1522-1528 (2013).

2. Tardif, J. C. et al. N. Engl. J. Med. 381, 2497-2505 (2019).

3. Tardif, J.-C. et al. Preprint at medRxiv https://doi, org/10.1101/2021.01.26.21250494 (2021).

4. Weng, J.-H. et al. Nat. Metabol. https://doi.org/10.1038/s42255021-00366-y (2021).

5. Lockhart, S. M., Saudek, V. \& O'Rahilly, S. Endocr. Rev. 41, 610-642 (2020).

6. Bauskin, A. R. et al. Cancer Res 65, 2330-2336 (2005)

7. Abd El-Aziz, S. H., Endo, Y., Miyamaori, H., Takino, T. \& Sato, H. Cancer Sci 98, 1330-1335 (2007).

8. Olsen, O. E., Skjærvik, A., Størdal, B. F., Sundan, A. \& Holien, T. PLoS ONE 12, e0187349 (2017).

9. Kempf, T. et al. Nat. Med. 17, 581-588 (2011).

10. Santos, I. et al. Proc. Natl Acad. Sci. USA 117, 12281-12287 (2020).

\section{Competing interests}

S.O.R. is an employee of the University of Cambridge and has provided remunerated consultancy services to the following pharmaceutical and biotechnology companies: Pfizer, AstraZeneca, Novo Nordisk, GSK and ERX. S.M.L. has no relevant conflicts of interest to declare. 